\title{
Assessing the Performance of an Integrated Health Information Exchange System Using Unstructured Supplementary Service Data
}

\author{
Adekunle, Y.A., Odunayo Yewande., Alao, O.D., Agbaje, M.O., Ebiesuwa Seun
}

\begin{abstract}
As budding nations gradually adopt the use of IT services to store medical records, several healthcare facilities are still unable to exchange information because of limited access to high speed internet service and high cost of operations. Established HIE implementations are designed to rely primarily on such tools as the internet. As a result, healthcare facilities in budding nations cannot embrace HIE to the maximum to accomplish interoperability. A versatile and flexible HIE system was developed in this research to accomplish exchange of health information. The system adapted the technique for direct exchange of HIE. This technique uses the Unstructured Supplementary Service Data (USSD) as the medium for transmitting encrypted HL7 version 2.X encoded messages adopting the Signaling System 7 (SS7) exchange protocol. The system was tested by establishing and studying a normal situation of communication between two health facilities that were interacting. The information recorded included the quality of the message delivery, the length of the communication and the precision of the contents of the transmitted message. In conclusion, the findings indicate a process that relays health information of patients in Internet-deprived regions in an effective and safe way. This idea of transmitting encrypted cryptic messages through USSD can also be applied to several other fields of study, where the sharing of information is restricted by poor Internet service availability.
\end{abstract}

KEYWORDS- Health Information Exchange, Unstructured Supplementary Service Data, Interoperability, Signaling System 7

Manuscript received March 20, 2020

Adekunle, Y.A, Computer Science Department,

Babcock University, Ilishan-Remo, Nigeria

Odunayo Yewande, Computer Science Department,

Babcock University, Ilishan-Remo, Nigeria

Alao, O.D, Computer Science Department, Babcock University, Ilishan-Remo, Nigeria

Agbaje, M.O, Computer Science Department, Babcock University, Ilishan-Remo, Nigeria

Ebiesuwa Seun, Computer Science Department, Babcock University, Ilishan-Remo, Nigeria

\section{INTRODUCTION}

For several centuries, healthcare facilities have employed paper-based approach of storing their patients' medical reports. Many developed countries (like Norway, The United Kingdom and The United States of America) have adopted the electronic means of storing patient records for over twenty years which have yielder positive results and this has led to researchers digging further to enable optimal access of these patients' information, in case of emergencies [5].These medical errors may show up in diverse forms and some of these errors at some point in emergencies happen during the paramedics' handover or exchange to the health facility. An unsuccessful handover is regarded to as a collapse in dissemination of health information which in turn may have deadly effects on patient care and safety, including; misdiagnosis or unnecessary delays in diagnosis [8]. Crucial hindrances hindering equal healthcare information access, specifically in developing nations include technological proficiency, visibility, economics, language barrier, cultural differences, copyright and internet accessibility. This houses the obligation for information to be acceptable irrespective of the cultural background and pertinent within distinct settings and territories [12]. Study has proven that nine pertinent characteristics are crucial for an HER execution to have enviable value. They include: scalability, simplicity, the capability to take advantage of already available ICT infrastructure, the adoption of an exchange protocol that assures message relaying, imaging ability, the adoption of an integrated interface as the platform for exchange, the capability to be equivalent to incoming messages with available patients' information, the integration of patients' approval, privacy/restricted access and the adoption of standardization [1]. Budding nations should not be cut off from benefitting from access to patients' data and information, just because of the meager availability of the resources needed unlike her developed equal. [6] in their journal publication on the "Lack of Interoperable Health Information Systems in Developing Countries" accentuated the following as the main problems.

1. The blueprint of the HER application appears to be innovation and technology driven and does not comply with the established norms and models for portability and mobility. 
2. The above downside leads into a structure that is majorly internal with the major aim of following patients up and giving close monitoring.

3. Another issue is the absence of varying function ability which include, but not limited to addition of clinical resolution aid.

4. Going forward, the outcome also implies that present age of EHRs dearth conformity with open standards hampering and hindering characteristics of workability, federation, interoperability and transparency.

In 2012, HEAF conducted a study to establish if there was any proof of surviving National eHealth constituents within each of the crucial categorization: Basic eHealth Information structure, Governance and country possession, Basic ICT substructure and Health process domain elements. A trial was made to establish whether for every of the above listed quad, there was proof of designing and execution. HEAF made a record that there was lean proof that EHRs are adapted to aid patient care; they are majorly adopted in public health, important registration and illness observation. It majorly found program-precise electronic aid for patient minding e.g. Tuberculosis minding. Designing and executing of basic primary eHealth information structure depicted the lowest proof. 50\% of the nations assessed had no proof that problems of quality measures, country registries, medical data exchanges, interoperability and data storages are being looked into at all. The report also showed that Kenya is notably the only African nation with representation on the International Standard Organization (ISO) Technical Committee for Health Informatics ISO TC 215.

The recent problems and prospects in Africa's HIS were scrutinized in a 2013 research on Digital Health Information System in Africa's material and resource deprived nations. The study posited that major advancements have been noticed in the application of HIS as the years rolled by. Closing the HIS gap in these materials deprived African nations still pose issues, although the African HIS was distinguished to have enormous prospective.

The Medical Information Section was also said to be poorly developed, under-resourced, clumsy and shambling. Considerable sluggishness in accepting and adopting the speedily developing digital automation was pointed out in various parts of Africa. This drawback has been increased by skyrocketing technology amounts and the scarcity in the accessibility of skilled technology individuals and useful and significant software, so also the doubtfulness in making funding of health infrastructures a major concern in different African nations. This research also accentuates the essence for improved direction of the capability to distribute and pass on information in order to furnish the healthcare givers with necessary information [10].

Shannon et al. [11] information theory asserts that linear communication should comprise of an information source, a sender and encoder, a message, a transfer medium, a recipient, a decoder, and an endpoint [11]. Some literature theories perform as the spine of the UHealth HIA option. The theories are predominantly based on what to concentrate on to gain access and the USSD's capacity as an access and transfer instrument for information.

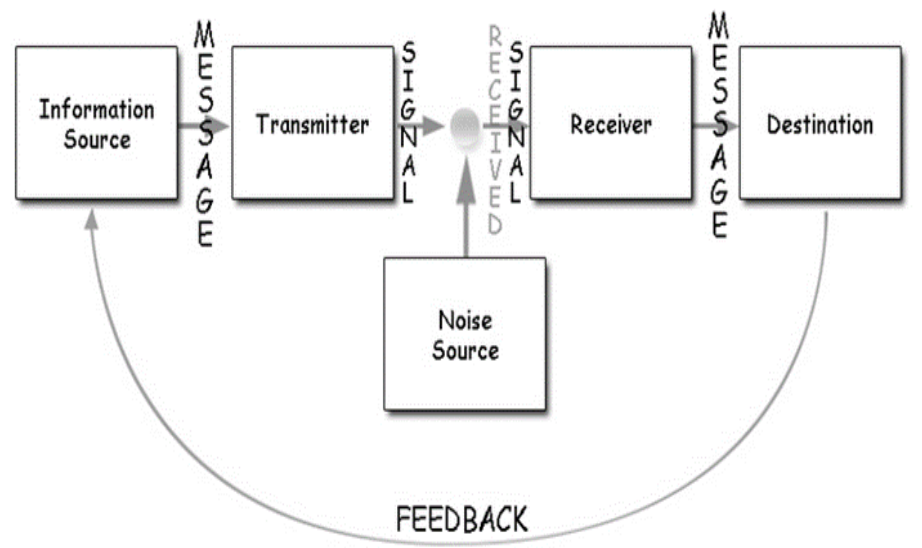

Fig 1: A linear communication model [11]

The theories are;

1. "The degree of lexical interoperability depends on the degree of agreement between the sender and recipient on the terms and the approval of the archetypes and models to be used" [4]. The semantic accessibility provided by the USSD / HIA model has been specified by the HL7 FHIR level 2 Step 2 which sets out stringent rules for the layout of exchange messages resolution, approval and exchange protocols.

2. "By aligning the features and specifications of the task at hand, people choose communication media" [9].The Social Influence Theory (SIT) stemmed from the fact that the Media Richness Theory (MRT) and the Social Presence Theory (SPT) were obsolete because not all facets of Computer Mediated Communications (CMC) were considered. MRT and SPT developed without direct scrutiny of new media and failed to recognize the suitability of SMS in distributed institutions, some of which include: no procedure for weighting richness scores is defined; and electronic mail offers richness requirements exempted from MRT, including things like various address capabilities, speed, convenience of use, individual experience, geographical dispersion of persons within communications networks (distributed organizations), and career pressure effect. This led to the development of the Social Influence Technology (SIT) and while SIT captured the CMC's main aspects, SIT does not really clarify why an individual selects one medium over another.

3. Alavi et al.[3] argued against MRT and their presumption that text-based communication technologies could be seen as lean media. They contended that the descriptive and predictive validity of this hypothesis has been reconsidered by new media. They suggested the Channel Expansion Theory (CET) using the SIT and posited that channel richness is also affected by the technique's social context, where expectations of media transitions are once again invested with the platform and other people using it. The CMC's complete capabilities have been taken into account and the USSD is one of the CMC's new communication media this utilized the 3-dimensional 
Interactivity cube of [7]. The nature of the HL7 FHIR messaging system inspired the preference of using USSD media. This system supports bidirectional communication system that is written in a distinct format and can be distributed via the USSD network concerned. In this situation, USSD came in handy because it is typically an easy tool to use that doesn't take so much work that participants don't need to be connected to the internet.

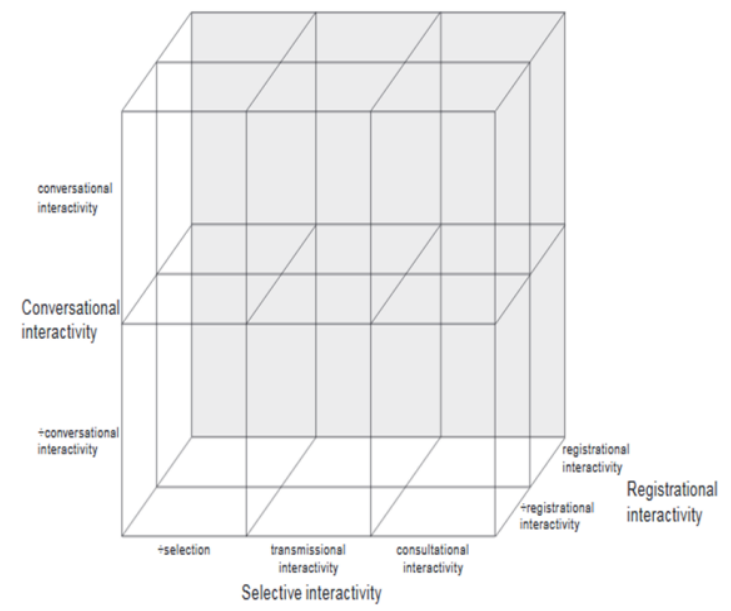

Fig 2: The 'Cube of Interactivity': a 3-dimensional Representation of the Dimensions of Interactivity

[7]

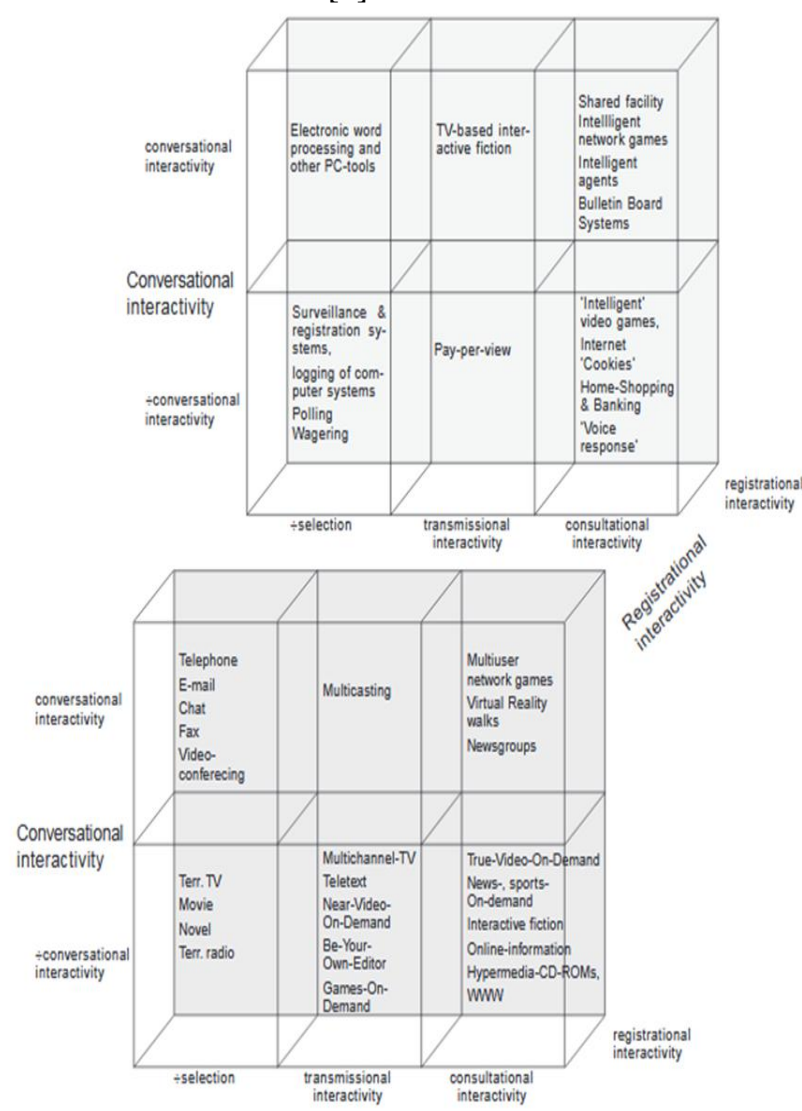

Fig 3: The 'Cube of Interactivity': a 3-dimensional Representation of the Dimensions of Interactivity [7].
When the systems work together in an easy way with little or no effort, and with some initiative in complicated ways, the two systems can be said to be interoperable. In either case, this effort does not need significant cooperation between various parties (to achieve technological interoperability). [8]. Two communication systems using the UHealth HIA model will interact with little effort in a straightforward manner, without the need for multi-stakeholder coordination.

1. Decomposition Theory of planned Behavior (TPB) [13] demonstrated that a deeper understanding of the partnership between the structures of belief and the background of intent necessitates the decomposition of attitudinal beliefs. [6] argued that cognitive elements of belief could not get arranged into a unitary cognitive component of belief. [13] have stated that the attitudinal assumption, based on the diffusion of innovation theory, has three outstanding features of an invention that affect acceptance as relative advantage, complexity and functionality [5]. Taylor et al.[13] demonstrated that the TPB's decomposed version has greater explaining capacity than the pure TPB and TRA versions. This is much needed as the UHealth HIA technology has to be put to test and this will come in handy.

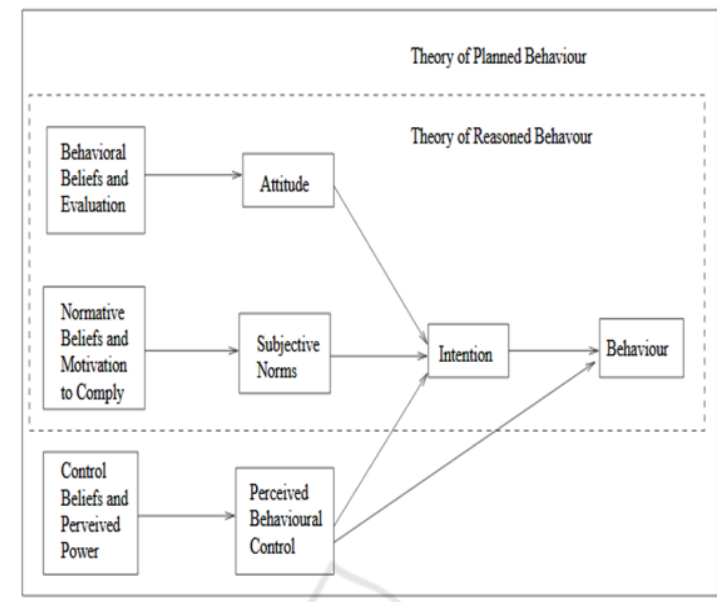

Fig 4: Theory of Reasoned Behavior model [13]

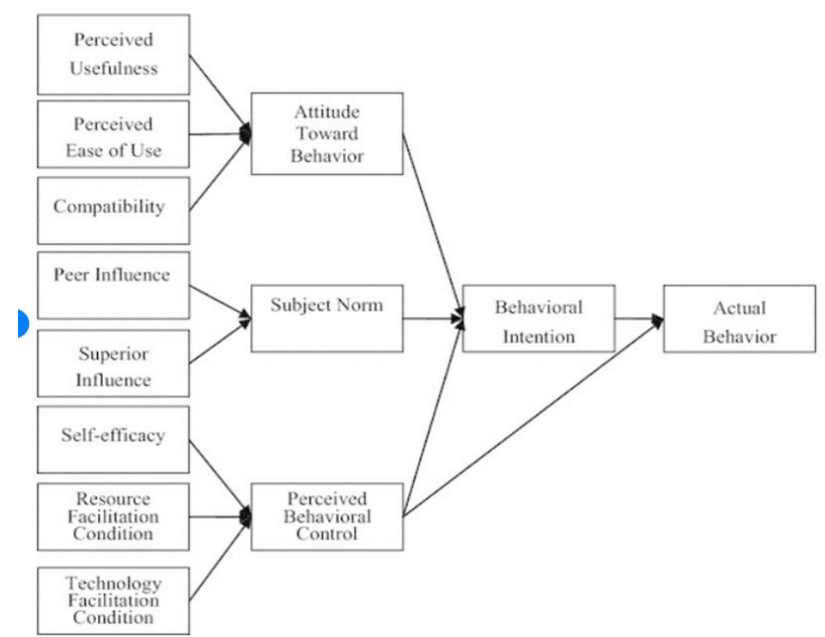

Fig 5: Theory of Planned Behavior model [13] 


\section{METHODOLOGY}

An evaluation was performed by simulating a hypothetical HIE situation, which was set up between two personal computers. This allows an exchange quality measurement along the following benchmarks:

- Delivery Affirmation;

- total exchange period;

- accuracy of the received message content and

- persistence of received local database information for future retrievals.

A detailed examination of the size of the information content to be accessed via USSD was carried out based on the 184-character content limit of a standard USSD message. This examination takes the segment and concerned field needed by an implementation of the UHealth model shown in Figure 1 into consideration. The HL7 ORU_R01 message was adopted for this examination because that is the message type which is commonly used for requesting and for responding to laboratory observations which often exchanged.

Table 1: Analysis of the USSD capacity and the HL7 v2.X encoding

\begin{tabular}{|c|c|c|c|}
\hline $\begin{array}{l}\text { Requi } \\
\text { red } \\
\text { Segme } \\
\text { nts }\end{array}$ & $\begin{array}{l}\text { Segment } \\
\text { Componen } \\
\text { ts }\end{array}$ & $\begin{array}{ll}\text { Required } & \text { Character } \\
\text { Constituents } & \end{array}$ & $\begin{array}{l}\text { Estimated } \\
\text { No.of } \\
\text { Characters }\end{array}$ \\
\hline \multirow{6}{*}{ MSH } & \multirow{4}{*}{$\begin{array}{l}\text { Required } \\
\text { Fields: }\end{array}$} & $\begin{array}{ll}\text { MSH-1 } & \text { (Delimeter } \\
\text { Characters) } & \\
\end{array}$ & 5 \\
\hline & & MSH-8 (Message Type) & 13 \\
\hline & & MSH-9 (Message Type) & 4 \\
\hline & & MSH-11 (Version) & 3 \\
\hline & \multirow[t]{2}{*}{ Delimeters } & Field Separator & 11 \\
\hline & & Component Separator & 2 \\
\hline \multirow{5}{*}{ PID } & \multirow{3}{*}{$\begin{array}{l}\text { Required } \\
\text { Fields: }\end{array}$} & PID-5 (Patient Name) & 35 \\
\hline & & PID-7 (Date of Birth) & 8 \\
\hline & & PID-8 (Sex) & 1 \\
\hline & \multirow[t]{2}{*}{ Delimeters } & Field Separator & 8 \\
\hline & & Component Separator & 2 \\
\hline \multirow{6}{*}{ OBR } & \multirow{4}{*}{$\begin{array}{l}\text { Required } \\
\text { Fields: }\end{array}$} & OBR-1 & 1 \\
\hline & & OBR-3 & 15 \\
\hline & & OBR-4 & 2 \\
\hline & & OBR-25 & 3 \\
\hline & \multirow[t]{2}{*}{ Delimeters } & Field Separator & 25 \\
\hline & & Component Separator & 3 \\
\hline \multirow[b]{3}{*}{ OBX } & \multirow{2}{*}{$\begin{array}{l}\text { Required } \\
\text { Fields: }\end{array}$} & OBX-2 & 2 \\
\hline & & OBR-5 & 15 \\
\hline & Delimeters & Field Separator & 5 \\
\hline \multicolumn{3}{|c|}{ Characters } & 158 \\
\hline
\end{tabular}

Justification for the UHealth Model's Components: This study argues that what is important when it comes to interoperability is that the information is accessible when needed. In order to achieve interoperability between communicating systems, it is not relevant that information of all patients should be stored consistently; the essential thing is that the desired information should be available upon request.

Consequently, the request-response technique has been incorporated in the UHealth model design. Such that, a request was first made, and in reaction to the request, a response was provided. The core intention for the USSD approach was that there is a prevalence of existing mobile telecommunications networks in regions where Internet accessibility is not available, allowing the capability to send and receive USSD messages [10].

Technical interoperability is primarily concerned with the usable transmission of medical information via a secure communication media from a sender to a receiver. Considering that Internet connectivity in most hospitals in developing countries is often not readily available. The developed model addresses the possibility of using USSD to transmit Patient Information, as against the conventional internet-reliant health information shared in advanced countries, the developed model gives ground for exchange even when Internet connectivity is unavailable.

The USSD Technology choice was opted for as opposed to the dependability of internet connections due to its higher reliability in information accessibility in budding nations [10]. The Nigerian Ministry of Health records that "Applications based on USSD generally require minimum effort in terms of adaptability, scope and ease of adaptation" [12]. The designed model rides on the established HL7 version 2.6 standard to achieve semantic compatibility between interacting EHRs, in addition to functional interoperability. With the HL7 v2.6 in place, the accessed messages can be correctly interpreted according to the sender's purpose. "HL7 standards are the healthcare industry's most effective communications standards" [3].

Prototype Implementation using the UHealth System: A prototype software to function as the HIA software was designed to test the idea introduced in the model. The prototype was developed according to model UHealth. In conformity with standard software engineering practices, the Agile software development cycle model was used for the software development. The Agile software development includes diverse software development strategies under which specifications and solutions emerge through the joint effort of self-organizing and cross-functional teams and their end users. This supports proactive preparation, evolutionary growth, early implementation, and continuous improvement, and facilitates quick and flexible reaction to change.

The development of the prototype consisted of:

- UML case analysis was beneficial in demonstrating the usage requirements for the various actors in a casual and friendly way, and in showing the path of operation to achieve the user objective. UML state transition diagram employed to model the various 
states where a HIA message will transit through, from the start of the information sharing to the termination of the process.

- The model of the software prototype's internal components and interactions was achieved using an Object-Oriented modeling method using UML Class diagram, Database design and a four-tier architecture. The class diagram illustrates the system's static structure with objects, attributes, operations and relationships, while the database architecture summarizes the tables of the database and their relationships.

- Afterwards, the prototype coding was done according to the study and design. The HIA software was developed using Java J2EE. The database is specifically designed to avoid any inconsistency in changes, thus separating groups into separate tables in the database. The Advanced Encryption Standard (AES) was adopted for module encryption implementation. The AES was adopted because it allows ease of use for the USSD as it is a 2-way module.

- Deploying the prototype on Personal Computers to further evaluate core performance metrics.

UML Analysis of the Software Prototype- State Diagram: the figure below gives a depiction of the transition states of the HIE message within a system built with the UHealth model.

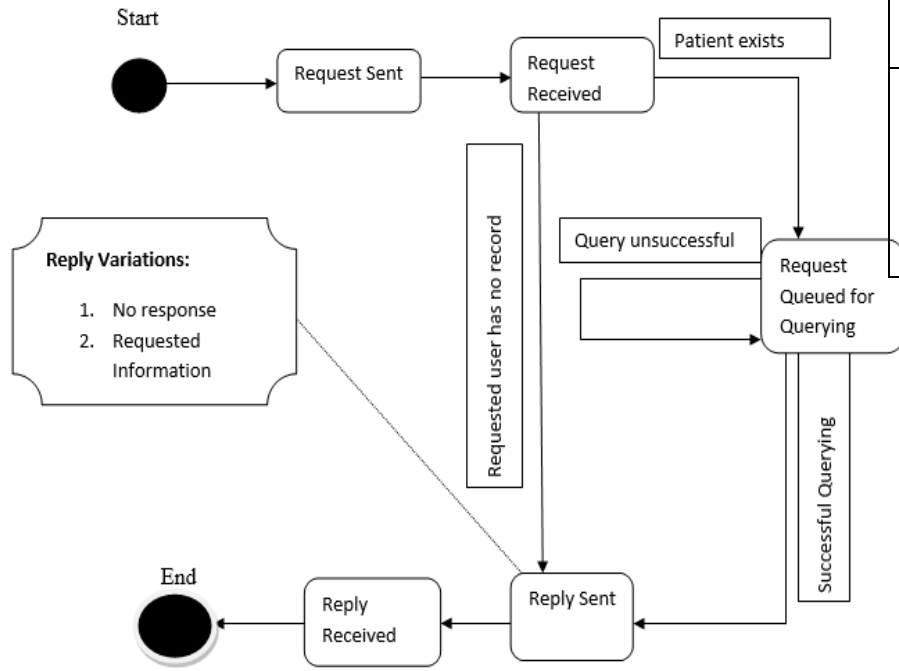

Fig 6: IHIE Uhealth Message state transition within the system

Based on the model, an HIE message transmits between 5 states (Request Sent; Request Received; Request Queued for Querying; Reply Sent; Reply Received). Table 2 briefly explains the different transitions, with the different reply variations of the "Reply Sent" state highlighted in $(\mathrm{S} / \mathrm{N} 2$ and 5).
Table 2: HIE Transition States

\begin{tabular}{|c|c|c|}
\hline $\mathbf{S} / \mathbf{N}$ & $\begin{array}{l}\text { Transition } \\
\text { Option }\end{array}$ & Details \\
\hline 1. & $\begin{array}{l}\text { "Request Sent" } \\
\text { "Request } \\
\text { Received" }\end{array}$ & $\begin{array}{l}\text { This first transition occurs as } \\
\text { soon as the request is successful } \\
\text { and the database has being } \\
\text { updated }\end{array}$ \\
\hline 2. & $\begin{array}{l}\text { "Request } \\
\text { Received" } \\
\text { "Reply Sent" }\end{array}$ & $\begin{array}{l}\text { This occurs when the request } \\
\text { receiver actor makes the move to } \\
\text { confirm request and there is no } \\
\text { match in the database. What } \\
\text { simply happens is that no } \\
\text { response is given. }\end{array}$ \\
\hline 3. & $\begin{array}{l}\text { "Request } \\
\text { Received" } \\
\text { "Request } \\
\text { Queued for } \\
\text { Querying" }\end{array}$ & $\begin{array}{l}\text { This occurs after confirming that } \\
\text { the patient exists in the database }\end{array}$ \\
\hline 4. & $\begin{array}{l}\text { "Request } \\
\text { Queued for } \\
\text { Querying" } \\
\text { "Reply Sent" }\end{array}$ & $\begin{array}{l}\text { This transition occurs when the } \\
\text { requested information is being } \\
\text { queried and there are matching } \\
\text { details in the database. This is } \\
\text { successful and the response is } \\
\text { displayed. }\end{array}$ \\
\hline 5. & $\begin{array}{l}\text { "Reply Sent" } \\
\text { "Reply } \\
\text { Received" }\end{array}$ & $\begin{array}{l}\text { This last transition occurs as soon } \\
\text { as the response sent is } \\
\text { successfully delivered to the } \\
\text { requesting side. }\end{array}$ \\
\hline \multicolumn{3}{|c|}{$\begin{array}{l}\text { The start state of an HIA message is the "Request Sent" } \\
\text { which takes form as soon as the actor requesting for } \\
\text { information clicks the send button, while the end state is } \\
\text { "Reply Received" which occurs as soon as the requesting } \\
\text { body receives a response message to an initial request. } \\
\text { Given that an HIA can be in only one state at a particular } \\
\text { instance, transition into different states is determined by the } \\
\text { outcome of the operation in the preceding state. }\end{array}$} \\
\hline
\end{tabular}

\section{REPORT}

The designed IHIE_Uhealth system is was tested using the Kapptivate online robot tool and performance was evaluated based on the following metrics; quality of quality of message delivery, the length of communication and the precision of the contents of messages transmitted.

\section{A. AVERAGE RESPONSE}

Time: Average response is the time of communication between the system and the database. Figure 1.7 shows the average response time for 100 clients requests. 


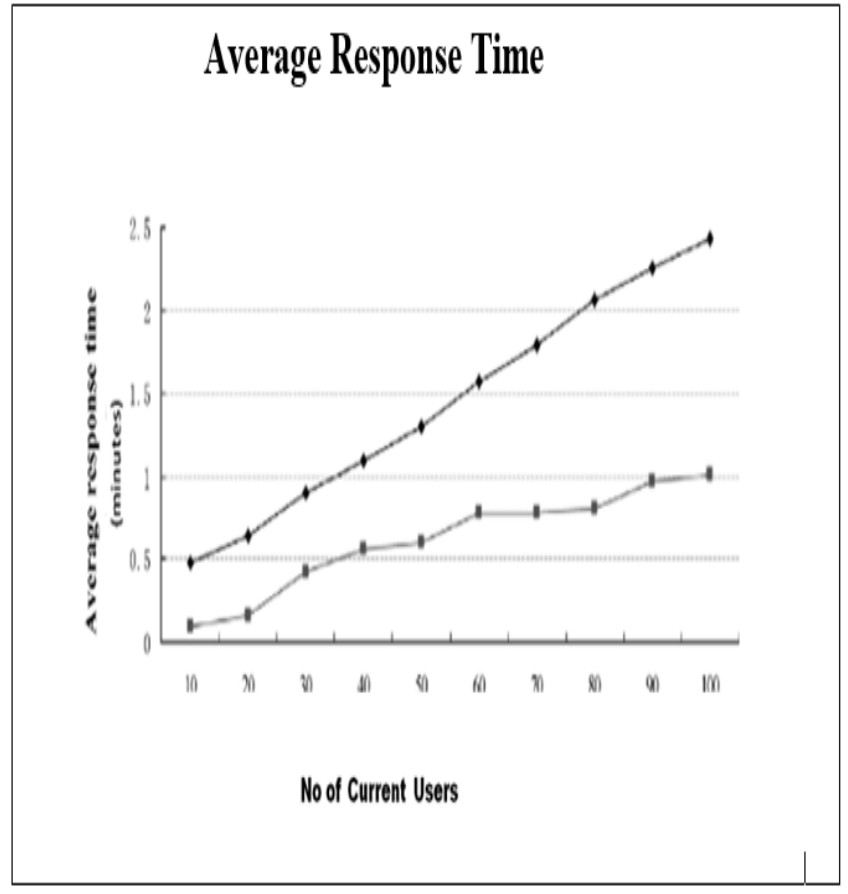

Fig 7: Average Response Time as against Number of current users

\section{B. BENCHMARKING}

The IHIE_Uhealth system was benchmarked against the SMS_HIE system designed by [1]. The SMS_HIE system proposed a model that allows health care organizations exchange patients' health information among themselves using USSD while the researcher's system proposed Health Information Exchange using USSD. The Average response time of the SMS_HIE was measured at 7 minutes while the IHIE_USSD average response time was measured at 2.5 minutes.

\section{CONCLUSION}

The main objective of this work was to create an opportunity for Hospitals in resource challenged areas to access Patients' health information when need be without relying on Internet connection. The specific objectives were to design the Health Information Access model using the USSD technology and implement and evaluate the performance of the designed model.A model was thereafter designed to make use of the USSD service coupled with HL7 v2.X message encoding structure to achieve technical and semantic accessibility. The prototype implementation of the designed model required the creation of a Web Service for reading and sending encrypted HL7 messages at intervals and a JAVA runtime environment User Interface. A performance evaluation was conducted on the prototype along the following benchmarks to assess reliability: Delivery Assurance, Total Period of Accessibility, and Persistence of received information for future retrievals on the local database.

As more hospitals in the implementation strategy and frameworks used for software solutions get on the "train of diversity," the challenge of integrating such diverse structures becomes more obvious and complicated. There is a need for cooperation between all hospitals to allow a national interoperability of information systems for health care. This partnership will require a significant participation of stakeholders in the health sector of each nation. Given the performance of the prototype HIE Software, the UHealth HIE model has proved effective and reliable. While considering implementing a software solution for information between developing countries, the quality of Internet connection is often a matter of concern. The UHealth HIE model is an alternative that can be explored to provide remote access to specific patient information at a lower cost and with high reliability to allow technology transfer in diversified regions. The model was developed with the salability versatility in mind and as such it can be easily adapted to cover more facets of eHealth and other sectors in resource-challenged nations. It cannot be ruled out that this research provides for relevant patient information to be made available to hospitals. The average time needed for accessing messages is competitive this would be better than spending time and money conducting laboratory tests and diagnostic procedures.

The research contribution needs the participation of a Mobile Network Operator and the involvement of the Federal Ministry of Health to achieve the maximum benefit of implementing the UHealth HIE system. A serious attention must be given to the enabling technologies currently in place in hospitals by finding solutions that require minimal cost implications-as most hospitals in resource-challenged nations are working on lean budget allocation for software package integration.

The implementation of the UHealth HIE model for Health Information Access in Nigeria can best be introduced by developing a caller community with a wide reach of any one of the nation's Mobile Network Operator. This will protect the monetary cost of engaging USSD services between hospital contacts to an affordable monthly subscription. This way it can adopt a systematic approach to evolution and development, giving room for process growth and change.

\section{REFERENCES}

[1] Akinde, A. (2016). A model for health information exchange using SMS as alternative transfer media. Asian Journal of Computer Science And Information Technology , 35-41

[2] Alali, H., \& Alweshah, M. (2014). Hospital Information Systems Success: Towards an Evaluation Framework. Australian Journal of Basic and Applied Sciences , 8(9), 45-51.

[3] Carlson, J. \& Zmud, R. (1999). Channel Expansion Theory and the Experiential Nature of Media Richness. The Academy of Management Journal , pp. 153-170, https://doi.org/10.2307/257090.

[4] CEN/ISSS. (2005). Current and future standardization issues in the eHealth domain: Achieving Interoperability. CEN/ISSS

[5] Fraser, H., Biondich, P., Choi, D., Mamlin, B., \& Szolovits, P. (2005). Implementing electronic medicl record sytems in developing countries. Informatics in primary cure, 83-95 
[6] Gambo, L., Oluwagbemi, O., \& Achimogu, P. (2011). Lack of Interoperable Health Information Systems in Developing Countries: An impact analysis. Journal of Health informatics in developing Countries , 5(1).

[7] Jensen, J. (1998). 'Interactivity' Tracking a New Concept in Media and Communication Studies semantic scholar. Retrieved from https://pdfs.semanticscholar.org/b04d/e1353d87619b 40 102fcab04f393615ba4e10.pdf

[8] Kachalia, A., Gandhi, T., Puopolo, A., Yoon, C., Studdert, D., Richard, R., Eric, T. \& Troyen, B. (2007). Missed and Delayed Diagnoses in the Emergency Department: A Study of Closed Malpractice Claims From 4 Liability Insurers. Annals of emergency medicine , 49(2): 195-205.

[9] Marin, D. B., \& Minsky, B. D., (1999). Why faculty members use e-mail: The role of individual differences in channel choice. The journal of Business Communication, 36(2), 194.

[10] Pape-Hapgard. (2011). Transversal interoperability for interdisciplinarity in e-Health - A study of models needed for interoperability in the Danish health system. Medical Informatics Group, Department of Health Science and Technology. Aalborg University, Denmark. Retrieved from vbn.aau: http://vbn.aau.dk/en/publications-phd-healthsciences/ transversal-interoperability-for-interdisciplinary-in-eh ealth(c4bcb549-401f-4e43-adf9-a3ae143839c7)/expor t.html

[11] Shannon, C. E., \& Weaver, W. (1949). The mathematical theory of communication. Universiy of Illinois. University of Illinois Press. Retrieved from http://www.mast.queensu.ca/-math474/shannon 1948. pdf

[12] Shetty, A. (2011). TATA Docomo Introduces Facebook, Twitter Access via USSD. Retrieved from Firstpost.com:

https://www.firstpost.com/tech/news-analysis/tata-doc omo-introduces-facebook-twitter-access-via-ussd-358 6001.html

[13] Taylor, S., \& Todd, P. (1995). Understanding Information Technology Usage: A Test of Competing Models. Information Systems Research Journal, pp. 144-176. 